\title{
STUDY OF CONDUCTIVITY, OPTICAL CONSTANTS AND SOLID STATE PARAMETERS OF THIOUREA ZINC SULPHATE SINGLE CRYSTALS
}

\author{
Sandhya Ravi ${ }^{1}$, S.Chenthamarai ${ }^{2}$, R.Jayavel ${ }^{3}$ \\ ${ }^{1}$ Research Scholar, Research Department of Physics, SDNB Vaishnav College for Women, Tamilnadu, India \\ ${ }^{2}$ Associate Professor, Research Department of Physics, SDNB Vaishnav College for Women, Tamilnadu, India \\ ${ }^{3}$ Director, Centre for Nano Science and Technology, Anna University, Tamilnadu, India
}

\begin{abstract}
In the present paper single crystals of Thiourea Zinc Sulphate (TZS) have been subjected to conductivity studies, determination of optical constants and fundamental parameters. The dielectric constant and dielectric loss were used to calculate the AC conductivity of the grown crystals over a frequency range $50 \mathrm{~Hz}$ to $5 \mathrm{MHz}$ at temperatures $313 \mathrm{~K}, 323 \mathrm{~K}$ and $333 \mathrm{~K} \mathrm{respectively.}$ The activation energies for the conduction process were determined from the Arrhenius plots for different frequencies. The optical constants such as refractive index, reflectance and susceptibility of the TZS single crystals were evaluated from the Ultra violetVisible (UV-Vis) spectrum data. The Plasma energy, Penn gap energy, Fermi energy and polarizability of the grown crystals were estimated theoretically using the single crystal $X$-ray diffraction (XRD) data.
\end{abstract}

Keywords: Conductivity, Activation energy, Refractive index, Penn energy, Polarizability.

\section{INTRODUCTION}

Thiourea zinc sulphate (TZS) is a well known semi organic material with excellent NLO properties. It is a popularly demanded material in the fields of laser technology, optical communications, data storage technology and optical computing because of its high resistance to laser damage, high nonlinearity, wide transparency, low angular sensitivity and good mechanical hardness compared to many organic NLO crystals [1].

The single crystals of TZS have been grown from Thiourea and Zinc sulphate taken in an equimolar ratio (1:1) by slow evaporation solution growth technique at room temperature. Growth, single and powder X-ray diffraction studies, FTIR studies, UV-Visible studies, Dielectric studies and SHG analysis have been reported earlier by Sandhya et al [2]. The Dielectric constant and dielectric loss values have been used to determine the AC conductivity of the single crystals. The linearity of the Arrhenius plots has led to the determination of activation energy at different frequencies. The optical band gap energy obtained from the UV-Visible spectrum has been used to determine the refractive index of the TZS single crystals. The reflectance and susceptibility of the crystals have also been evaluated. The fundamental parameters such as Plasma energy, Penn energy, Fermi energy and polarizability have been estimated.

\section{CONDUCTIVITY STUDIES}

The AC conductivity of TZS single crystals were calculated with the formula,

$$
\boldsymbol{\sigma}_{\mathrm{ac}}=\mathbf{2} \boldsymbol{\pi} \boldsymbol{\varepsilon}_{\mathbf{0}} \boldsymbol{\varepsilon}_{\mathrm{r}} \tan \boldsymbol{\delta}, \text { expressed in mho } \mathrm{m}^{-1}
$$

Where $f$ is the frequency range of measurement, $\varepsilon_{0}$ is the permittivity of free space, $\varepsilon_{\mathrm{r}}$ and $\tan \delta$ are the measured values of dielectric constant and dielectric loss. The measurements were made using the HIOKI 3532-50 LCR HITESTER instrument over a frequency range $50 \mathrm{~Hz}$ to 5 $\mathrm{MHz}$ at temperatures $313 \mathrm{~K}, 323 \mathrm{~K}$ and $333 \mathrm{~K}$ respectively. The plot of $\ln \sigma_{\mathrm{ac}}$ vs. $\ln \mathrm{f}$ for temperatures $313 \mathrm{~K}, 323 \mathrm{~K}$ and $333 \mathrm{~K}$ is shown in Figure1.

The conductivity is found to increase with increase in frequency. This is because, the conductivity is proportional to the mobility and carrier concentration through the well known relation:

$$
\boldsymbol{\sigma}_{\mathrm{ac}}=\mathbf{n}_{\mathrm{d}} \mathbf{e} \boldsymbol{\mu}_{\mathrm{e}}
$$

where $\mu_{\mathrm{e}}$ is the mobility of electrons and $n_{d}$ the number density of electrons [3]. As frequency increases mobility of electrons increases and hence conductivity rises. In the region near $1 \mathrm{MHz}$, systematic minima values are observed. The reason for this may be, thiourea inclusion compounds in crystalline state generally form a commensurate lattice with guest molecules occupying preferred sites but are strongly orientationally disordered [4]. There is a general rise in conductivity with increasing temperature.

The plots between $\ln \sigma_{\mathrm{ac}}$ and 1000/T for frequencies $9 \mathrm{KHz}$, $60 \mathrm{KHz}$ and $5 \mathrm{MHz}$ were found to be very linear and are shown in Figure 2, Figure 3 and Figure 4 respectively. So the conductivity values can be fitted into the Arrhenius relation: 
where $E_{a}$ is the activation energy, $k$ the Boltzmann constant, $\mathrm{T}$ the absolute temperature and $\sigma_{0}$ a constant depending upon the material. The activation energies were evaluated from the slopes of the plots $\left(\mathrm{E}_{\mathrm{a}}=-\right.$ slope $\left.\mathrm{x} k\right)$. The activation energies for the $\mathrm{AC}$ conduction process for frequencies 9 $\mathrm{KHz}, 60 \mathrm{KHz}$ and $5 \mathrm{MHz}$ were $0.1719 \mathrm{eV}, 0.0936 \mathrm{eV}$ and $0.0252 \mathrm{eV}$ respectively. The activation energies decrease with increasing frequency. The increase of the applied frequency enhances electronic jumps between localized states, consequently the AC activation energy decreases with increasing frequency [5].

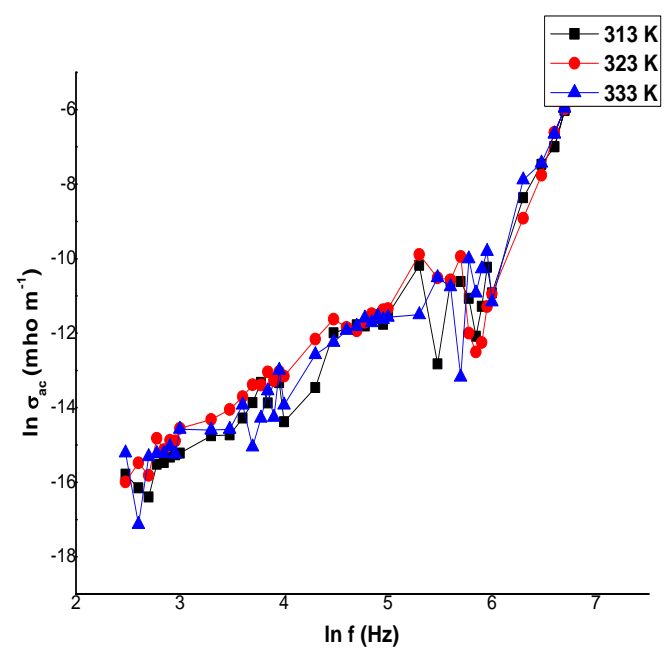

Fig 1: $\ln \sigma_{\mathrm{ac}}$ vs. $\ln \mathrm{f}$

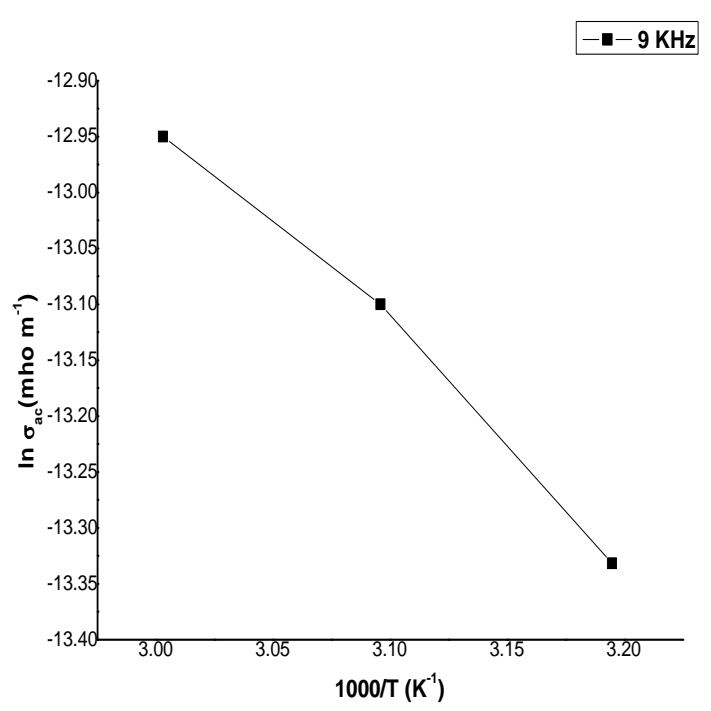

Fig 2: $\ln \sigma_{\mathrm{ac}}$ Vs. 1000/T for $9 \mathrm{KHz}$

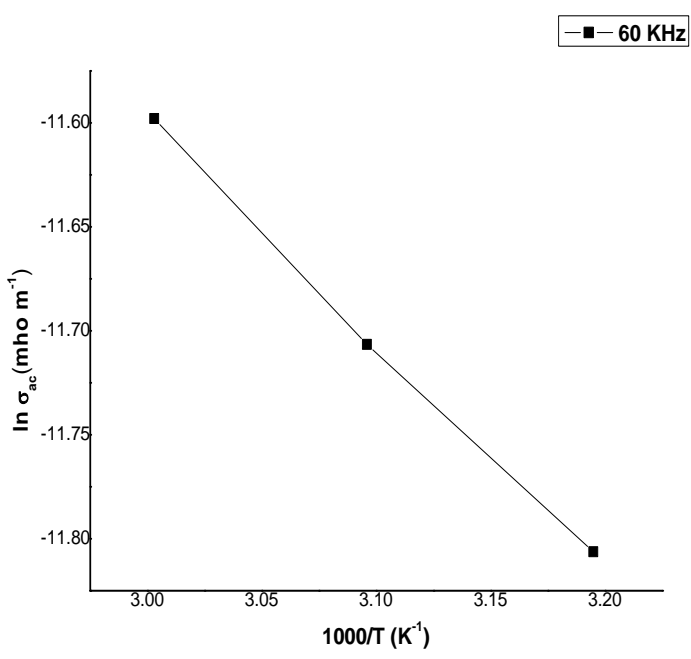

Fig 3: $\ln \sigma_{\mathrm{ac}}$ vs. 1000/T for $60 \mathrm{KHz}$

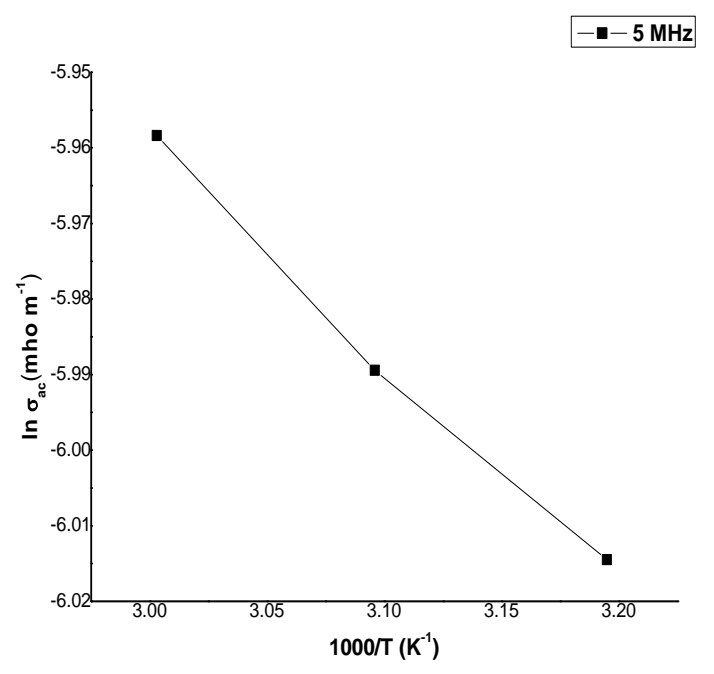

Fig 4: $\ln \sigma_{\mathrm{ac}}$ vs. $1000 / \mathrm{T}$ for $5 \mathrm{MHz}$

\section{DETERMINATION OF OPTICAL CONSTANTS}

The study of optical constants of a material is very important for NLO applications. The optical band gap energy of the single crystals of TZS has been reported earlier as $4.77 \mathrm{eV}$ from the UV-Visible spectrum [2]. The relation between optical band energy $\left(\mathrm{E}_{\mathrm{g}}\right)$ and the refractive index (n) of the crystal is $\mathbf{E}_{\mathrm{g}} \mathbf{e}^{\mathbf{n}}=\mathbf{3 6 . 3}[6]$. The calculated refractive index is then used to determine the reflectance(R) by applying the formula:

$$
R=\left(\frac{n-1}{n+1}\right)^{2}
$$

The refractive index and the reflectance of TZS single crystals were calculated as 2.03 and 0.115 respectively. The high value of the refractive index and the low value of reflectance reveal that the TZS crystals are more transparent 
to transmit light from $250 \mathrm{~nm}$ to above $800 \mathrm{~nm}$ [7]. The high transmission, low absorbance, low reflectance make the material an important one for anti-reflection coating in solar thermal devices and non linear optical applications [8]. The extinction coefficient $(\mathrm{K})$ was estimated using the equation:

$$
K=\frac{\alpha_{a} \pi}{4 \pi},
$$

Where $\alpha_{\mathrm{a}}$ is the absorbance which is obtained from the UVVisible spectrum [2]. The plot of extinction coefficient versus wavelength is shown in Figure 5. The extinction coefficient is found to decrease as the wavelength increases to a very low value up to $250 \mathrm{~nm}$ and thereafter remains constant. The low extinction coefficient is of vital importance for applications in information processing and computing devices [9]. The electrical susceptibility was calculated from the refractive index of the crystals according to the formula:

$$
X_{c}=n^{2}-1
$$

The susceptibility was determined to be 3.1209 which is larger than unity. This results in greater development of polarization in the presence of dc electrical fields, hence leading to greater mobility [10].

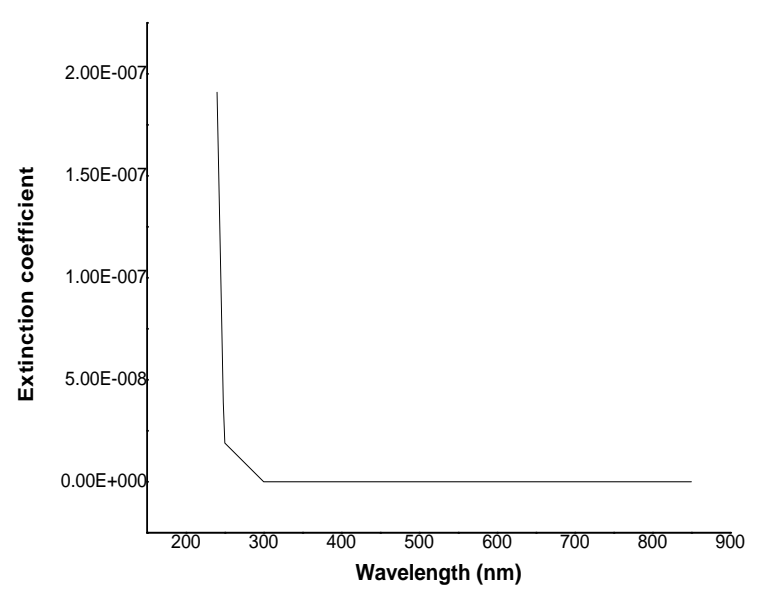

Fig 5: Extinction Coefficient vs. Wavelength

\section{EVALUATION OF SOLID STATE PARAMETERS}

The solid state parameters are necessary for analyzing the second harmonic efficiency of the TZS crystals. The valence electron plasma energy plays an important role in optical properties of NLO crystals. Generally light of frequency smaller than the plasma energy of the material are reflected on account of the screening effect of the valence electrons, while frequencies greater than the plasma frequency are transmitted due to lack of time for the electrons to respond [11]. The valence electron plasma energy of the TZS single crystals was evaluated by:

$$
\hbar \omega_{p}=28.8\left(\frac{Z \rho}{M}\right)^{1 / 2},
$$

Where $\mathrm{Z}$ is the total number of valence electrons in the crystal (56), $\rho$ is the crystal density $\left(0.293 \mathrm{gm} / \mathrm{cm}^{3}\right)$ and $M$ (237.59) the total molecular weight of the crystal, $\omega_{p}$ is the plasma frequency. The crystal density was calculated from [12]:

$$
\rho=\frac{M m_{a}}{V}
$$

where $\mathrm{m}_{\mathrm{a}}$ is the atomic mass unit and $\mathrm{V}$ volume of the unit cell of TZS single crystal which is obtained from the single XRD data [2]. The knowledge of Penn gap reveals the minimum energy required to excite an electron from the isotropic spherical momentum space of the covalently bonded TZS single crystal [13]. The Penn gap $\left(E_{P}\right)$ is calculated as follows [14]:

$$
E_{p}=\frac{\hbar \omega_{p}}{\left(\varepsilon_{\infty}-1\right)^{1 / 2}}
$$

where $\varepsilon_{\infty}$ is the high frequency dielectric constant (At 1 $\mathrm{MHz}) . \varepsilon_{\infty}$ at $1 \mathrm{MHz}$ as obtained from the dielectric study data [2] is equal to 16.2 .

The Fermi energy in terms of valence electron plasma energy is [14]:

$$
E_{F}=0.2948\left(\hbar \omega_{p}\right)^{4 / 3}
$$

The polarizability of the TZS single crystals is calculated using the formula [14]:

$\alpha_{p}=\left[\frac{\left(\hbar \omega_{p}\right)^{2} S_{0}}{\left(\hbar \omega_{P}\right)^{2} S_{0}+3 E_{p}^{2}}\right] \times \frac{M}{\rho} \times 0.396 \times 10^{-24}$

where $S_{0}$ is a constant given by

$$
S_{0}=1-\left[\frac{E_{p}}{4 E_{F}}\right]+\frac{1}{3}\left[\frac{E_{p}}{4 E_{F}}\right]^{2}
$$

The polarizability can also be evaluated from the Clausius Mossotti relation:

$$
\alpha_{p}=\left(\frac{3 M}{4 \pi \rho N}\right)\left[\frac{\varepsilon_{\infty}-1}{\varepsilon_{\infty}+2}\right]
$$

The solid state parameters are listed in Table 1.

Table 1: Solid state parameters of TZS single crystals

\begin{tabular}{|l|l|}
\hline Parameters & Value for TZS single crystals \\
\hline Plasma Energy (eV) & $7.568 \mathrm{eV}$ \\
\hline Penn Gap (eV) & $1.9411 \mathrm{eV}$ \\
\hline Fermi Energy (eV) & $4.3773 \mathrm{eV}$ \\
\hline
\end{tabular}




\begin{tabular}{|l|l|}
\hline $\begin{array}{l}\text { Polarizability by Penn } \\
\text { Gap }\left(\mathrm{cm}^{3}\right)\end{array}$ & $26.3005 \times 10^{-23} \mathrm{~cm}^{3}$ \\
\hline $\begin{array}{l}\text { Polarizability by by } \\
\begin{array}{l}\text { Clausius Mossotti } \\
\text { relation }\left(\mathrm{cm}^{3}\right)\end{array}\end{array}$ & $26.738 \times 10^{-23} \mathrm{~cm}^{3}$ \\
\hline
\end{tabular}

The polarizability of TZS single crystals is found to be 3.4 times higher than the polarizability of Tris Thiourea Zinc Sulphate (ZTS) single crystals [15].

\section{CONCLUSION}

TZS single crystals were grown by slow evaporation solution growth technique at room temperature. AC conductivity study of the grown crystals was carried out using the data from the dielectric analysis. The conductivity was found to increase with frequency as well as temperature. The activation energy for $\mathrm{AC}$ conduction was calculated and was found to decrease with increasing frequency. The optical constants of the TZS single crystals were evaluated from the UV-Visible spectrum data. The TZS single crystals were found to possess high transmittance, low absorbance, low reflectance, low extinction coefficient and high susceptibility. These optical characteristics make the TZS single crystals best suited for NLO applications. The Solid state parameters such as Valence electron plasma energy, Penn gap, Fermi energy and polarizability of the single crystals were estimated. The polarizability value determined using Penn gap agreed very well with the polarizability value obtained using ClausiusMossotti relation.

\section{REFERENCES}

[1]. G. Ramasamy and Subbiah Meenakshisundaram, Journal of Crystal Growth, 377, 197, (2013).

[2]. Sandhya Ravi and S.Chenthamarai, Indian Journal of Scientific Research, 9(1), 51, (2014).

[3]. T. Arumanayagam and P. Murugakoothan, Journal of Minerals \& Materials Characterization \& Engineering, 10(13), 1225, (2011).

[4]. Hanna Maluszynka and Piotr Czarnecki, Journal of Crystallography, 221, 218, (2006).

[5]. F. Yakuphanoglu, Y. Aydogdu, U. Schatzschneider and E. Rentschler, Solid State Communications, 128, 63, (2003).

[6]. R.R.Reddy and S. Anjaneyulu, Physica status solidi (b), 174, 91, (1992).

[7]. P. Vasudevan, S. Sankar and D. Jayaraman, Bulletin of the Korean Chemical Society, 34(1), 128, (2013).

[8]. F.Helen and G.Kanchana, Indian Journal of Pure \& Applied Physics, 52, 821, (2014).

[9]. R.N. Shaikh, Mohd.Anis, M.D. Shirsat and S.S. Hussaini, IOSR Journal of Applied Physics, 6(1), 42, (2014).

[10]. Laurence A.Belfiore, Physical Properties of Macromolecules (Hoboken, New Jersey, John Wiley and Sons Inc., 2010).

[11]. Glenn A. Burdick, Physical Review, 129, 138, (1963).

[12]. E.Prince, International Tables for CrystallographyVolume C- Mathematical, physical and chemical tables ( AA Dordrecht, The Neatherlands, Kluwer Academic Publishers, 2004).
[13]. D.R.Penn, Physical Review, 128, 2093, (1962).

[14]. N.M. Ravindra, R.P. Bharadwaj, K.Sunil Kumar and V.K. Srinivastava, Infrared Physics, 21, 369, (1981).

[15]. M. Sumithra Devi, A. P. Arthi, P. Sagayaraj and K. Thamizharasan, Archives of Physics Research, 4 (5), 67, (2013). 